\title{
Fertility and fragrance: another cause of Kallmann syndrome
}

\author{
Shlomo Melmed \\ Pituitary Center, Department of Medicine, Cedars-Sinai Medical Center, Los Angeles, California, USA.
}

\begin{abstract}
Kallmann syndrome is an inherited deficiency of gonadotropin-releasing hormone ( $\mathrm{GnRH}$ ) that is characterized by hypogonadism with delayed or absent puberty and dysfunctional olfaction. While Kallmann syndromeassociated mutations have been identified in some sets of patients, for many of these individuals, the underlying cause remains unknown. In this issue of the $\mathrm{JCl}$, Cariboni and colleagues identify mutations in semaphorin 3E (SEMA3E) in two brothers with Kallmann syndrome. In animal models, loss of SEMA3E signaling recapitulated phenotypes of the probands and resulted in enhanced $\mathrm{GnRH}$ neuron death during development. The results of this study offer important insight into the development of Kallmann syndrome and provide tools for elucidating mutations that underlie complex hormonal phenotypes.
\end{abstract}

\section{The hypothalamic/pituitary/} gonadal axis

Function of the hypothalamic/pituitary/ target gland axis is tightly regulated by three interacting tiers of control that integrate brain, anterior pituitary, and peripheral hormone signaling. The anterior pituitary gland comprises at least six highly differentiated cell types that each express a specific trophic hormone. Corticotroph cells express adrenocorticotrophin (ACTH), which regulates adrenal function; somatotrophs express growth hormone (GH), which regulates growth and body composition; thyrotrophs express thyrotrophin (TSH), which regulates thyroid function; lactotrophs express prolactin (PRL), which regulates lactation; and gonadotrophs express both luteinizing hormone (LH) and follicle-stimulating hormone (FSH), which regulate reproductive function (1). Synthesis and secretion of each of these trophic hormones are controlled by hypothalamic-releasing and/or-inhibiting hormones, which traverse the hypophyseal portal system to impinge on cognate $G$ protein-coupled anterior pituitary cell surface receptors. Hypothalamic corticotrophin-releasing hormone $(\mathrm{CRH})$ induces ACTH; GH-releasing hormone (GHRH) and somatostatin (SRIF) regulate GH; thyrotrophin-releasing hormone (TRH) and dopamine regulate PRL and TSH; and gonadotropin-releasing hormone $(\mathrm{GnRH})$ regulates FSH and LH synthesis and secretion. Within the pituitary, paracrine factors impinge directly on specific cell functions. Target-specific tissue hormones, including adrenal and gonadal steroids, insulin-like growth factor (IGF-I), and thyroid hormone, also participate in feedback loops to regulate their respective pituitary trophic hormone secretion (1).

Gonadal function is mediated by the maturation and function of germ cells as well as by sex steroid-dependent regulation of secondary sexual characteristics (2). During development, central GnRH neurons arise mainly from the olfactory placode and migrate to the hypothalamus, as determined by regulated neuronal and glial scaffolding as well as chemotactic and adhesion molecules (refs. 3, 4, and Figure 1).

The hypothalamic gene GNRH1 encodes a 92 -aa precursor protein that

Related Article: p. 2413

Conflict of interest: The author has declared that no conflict of interest exists.

Reference information: / Clin Invest. 2015;125(6):2275-2278. doi:10.1172/JCI82061. 


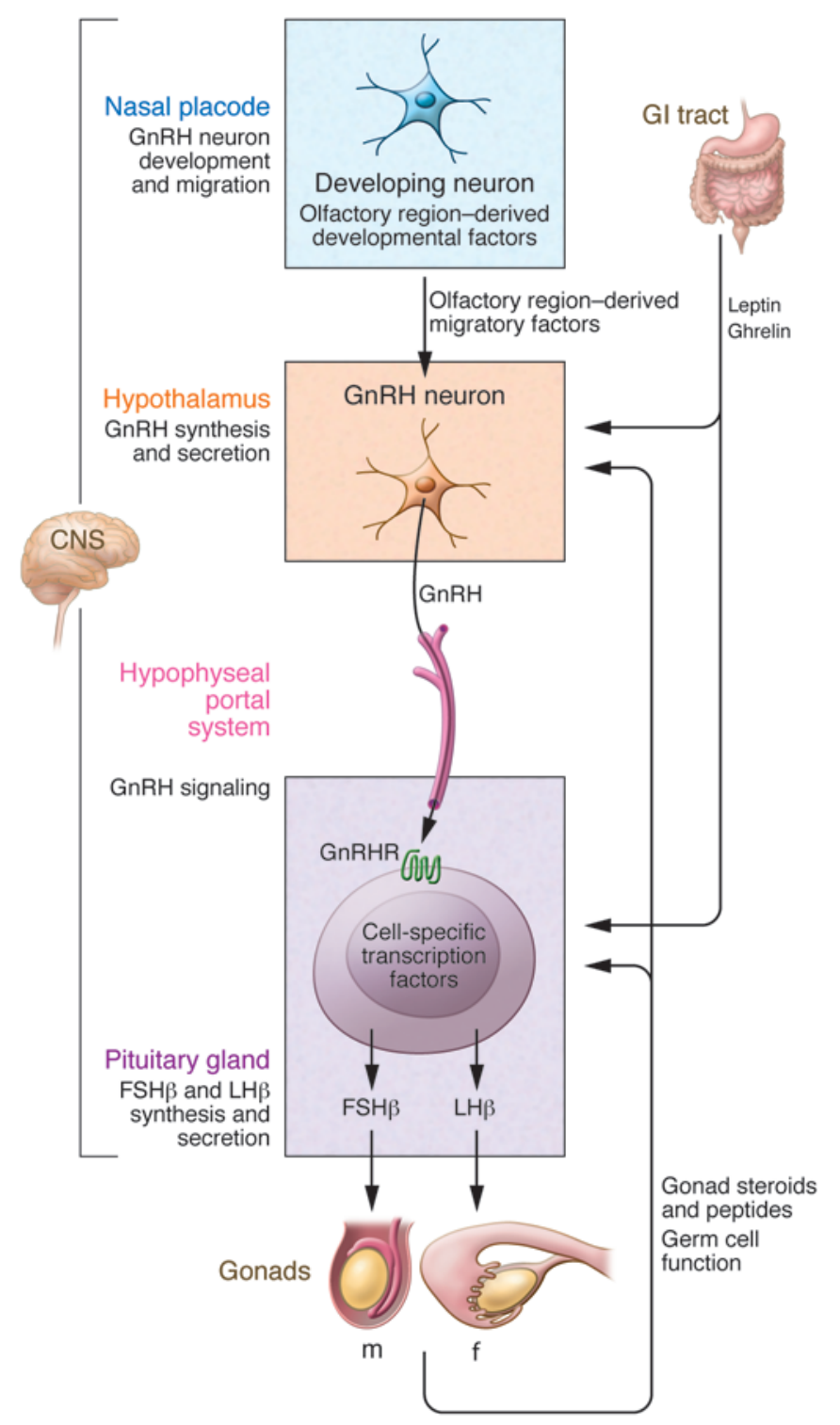

or secretion (12). GnRH secretion is also regulated by nutrition-related hormones, including leptin and ghrelin (13). GnRH action may be abrogated by mutations in the GnRH receptor, leading to trafficking or signaling defects. As GnRHR signaling specificity is strongly determined by conformational integrity, mutations that lead to receptor misfolding may disrupt hypothalamic signal transduction to the gonadotroph cell (14). Reinforcing this observation, defective GnRHR stability may be rescued by small-molecule chaperones (15). Within the gonadotroph, the distal component of the hypothalamic/pituitary cascade, LH and FSH synthesis, may be disrupted as the result of mutations in genes encoding pituitary hormones or gonadotroph-specific transcription factors. Acquired hypogo- nadotrophism may also be caused by sellar masses compressing gonadotrophs, or by structural damage after surgery, radiation, or pituitary inflammation (1).

\section{Mechanisms underlying Kallmann syndrome}

Kallmann syndrome is an X-linked disorder that results from disruption of hypothalamic GnRH neuron development and presents with associated olfactory nerve agenesis or hypoplasia, leading to hyposmia/anosmia (16). The syndrome comprises varying degrees of hypogonadism with attenuated pituitary FSH and LH secretion, as well as heterogenous features that are linked to specific mutations but are not associated with the reproductive system. For example, synkinesia is associated
Figure 1. The cascade of reproductive axis signal amplification initiated by $\mathrm{GnRH}$ and culminating in gonadal function confers fertility and secondary sex characteristics. Central molecules, including KAL1, FGF8/ FGFR1, PROK2/PROK2R, NELF, SEMA3A/ PLXND1, HS6ST1, CHD7, and WDR11, determine $\mathrm{GnRH}$ neuron development in the olfactory region (27) and migration to the hypothalamus. Multiple intrahypothalamic, nutritional, and hormonal signals, such as KISS1/KISS1R, TAC3/TAC3R, and PCSK1, determine the generation of age- and time-determined $\mathrm{GnRH}$ pulses, which traverse the hypophyseal portal circulation to signal through the pituitary gonadotroph GnRHR. Transcription factors, including NR5A1, NROB5, SF1, DAX1, and PROP1, also determine cell-specific gonadotropin hormone (FSH and LH) expression. Unique pulsatile FSH and LH release patterns induce gonadal hormones and determine germ cell function. Hypogonadotrophic hypogonadism can be caused by disruptions at each of the depicted CNS/hypothalamic/pituitary levels. Heterogenous oligogenic patterns of inheritance may be associated with a similar clinical phenotype of gonadal failure in the presence of inappropriately low gonadotropin hormone levels. Acquired causes of hypogonadotrophic hypogonadism include neoplastic, inflammatory, or iatrogenic (surgery, radiation) hypothalamic-pituitary damage.

with Kallmann syndrome 1 (KAL1, which encodes anosmin) mutations; hearing loss is linked to chromodomain helicase DNA-binding protein 7 (CHD7) mutations; and dental agenesis and syndactyly are associated with mutations in FGF $(10,16)$. Other clinical features of both autosomal dominant and recessive forms of Kallmann syndrome include color blindness, renal agenesis, and cryptorchidism $(16,17)$. MR imaging of individuals with Kallmann syndrome may also reveal hypoplasia in the olfactory tract.

As hypogonadotrophism is present throughout the lifespan of those with Kallmann syndrome, developing and mature tissues are deprived of gonadal steroid exposure, leading to hypogonadism with delayed puberty and attenuated secondary sexual development, including continued bone growth and primary amenorrhea in females, and micropenis in males (18). Moreover, these patients are devoid of hypothalamic GnRH and exhibit attenuated synthesis and secretion of LH and FSH by pituitary gonadotrophs, resulting in low or undetectable estradiol or testosterone secretion and atrophic gonads. 
Several factors have been identified that determine the development of olfactory placode cells in the developing brain and their migration toward the hypothalamus. Complex signaling enables GnRH precursor cells to navigate the frontal lobe, where they ultimately reside in the hypothalamic median eminence. Kallmann syndrome-linked mutations have been ascribed to dysfunctional $\mathrm{GnRH}$ neuron development, migration, survival, and differentiation. Anosmin is required for olfactory cell migration and adhesion (9), and loss of anosmin disrupts $\mathrm{GnRH}$ cell migration from the olfactory placode; however, this X-linked defect accounts for a only small cohort of these patients (2). Affected kindreds have also been shown to include individuals who manifest with autosomal dominant or recessive traits with variable penetrance. Heterogeneous combinations of mutations result in an oligogenic inheritance pattern and often include the gene encoding FGFR1 ligand, which regulates axonal development (19). Several gene products interact with the FGF network, including the chromatin-remodeling factor $\mathrm{CHD} 7$ (20). Mutations in CHD7 are a component of CHARGE syndrome, which includes coloboma, choanal atresia, and heart defects, along with growth and development dysfunction (21). Mutations of the gene encoding SEMA3A, which functions as an axonal pathway regulator of $\mathrm{GnRH}$ neurons, result in ectopic GnRH nasal neuron migration (22). Other factors, including neuronal migration factor NELF, WD repeat domain 11 (WDR11), and heparan sulfate 6-O-sulfotransferase 1 (HS6ST1), also participate in $\mathrm{GnRH}$ development or migration (18), and olfactory bulb development is regulated by prokineticin 2 (PROK2) (23).

Despite remarkable advances in identifying Kallmann syndrome-associated gene mutations, it is apparent that hitherto unidentified gene mutations underlie this syndrome in many patients with idiopathic phenotypes (24). In this issue, Cariboni et al. demonstrate that SEMA3E and the SEMA3E receptor PLXND1 are important factors for GnRH neuron survival in the developing brain after these cells have traversed the forebrain during hypothalamic migration. Using whole-exome sequencing and computational modeling, Cariboni and colleagues identify a SEMA3E mutation in two brothers with features of Kallmann syndrome (11). This particular missense mutation (R619C) enables selective death and apoptosis of central GnRHproducing neurons. SEMA3E, in conjunction with PLXND1, acts as a cell repellent and angiogenesis regulator (25) and is now shown by Cariboni et al. to function as a prosurvival factor for mature hypothalamic GnRH neurons by signaling through PLXND1 and its coreceptor $\mathrm{KDR}$, a kinase known to mediate neuronal growth. Whole-exome sequencing also revealed the presence of a CHD7 mutation in the two probands, indicating that the combination of heterozygous mutations underlies the disease, as fertility was enabled in both parents, each of whom harbored a heterozygous mutation. The presence of combined heterozygous CHD7 and SEMA3E mutations in the offspring afflicted with Kallmann syndrome is consistent with the proposed oligogenic pattern of Kallmann syndrome inheritance (26). Cariboni and colleagues also recapitulated the human phenotype in compound heterogenous mice that were null for both Plxndl and Chd7. GnRH neuron innervation in the median eminence was severely disrupted in these animals, further demonstrating the cooperative functions of SEMA3E and $\mathrm{CHD} 7$ in regulating $\mathrm{GnRH}$ neuron development (11).

SEMA3E was also shown to induce PLXND1-dependent P13K-mediated GnRH neuron survival, as GnRH neuron apoptosis was increased and $\mathrm{GnRH}$ neuron innervation was decreased in the developing brains of KO mice lacking either SEMA3E or PLXND1. This reduced adult median eminence population of GnRH-positive neurons recapitulated clinical features of hypogonadism, such as testicular atrophy. Accordingly, PLXND1 and CHD7 were proposed to synergistically regulate $\mathrm{GnRH}$ neuron development. As mechanisms underlying these observations likely do not involve indirect forebrain vascular effects nor reflect defective SEMA3E dimerization, Cariboni and colleagues propose that introduction of an extracellular cysteine may lead to disrupted protein structure (11).

\section{Conclusions and future directions}

The results of the study by Cariboni et al. (11) support adding SEMA3E and PLXND1 to the list of candidate genes to be considered when evaluating patients with hypogonadotrophic hypogonadism associated with hyposmia/anosmia. For patients with other as-yet idiopathic endocrine disorders, the approach of Cariboni and colleagues, which involved a combination of wholeexome sequencing, computational modeling, functional cell biology, and clinical recapitulation in $\mathrm{KO}$ transgenic mice, should lead to the discovery of additional genetic causes of complex, rare familial endocrine syndromes. Thus, the elegant discovery of Cariboni et al. sheds light on an investigational approach for further detection of as-yet cryptic causes of other central pituitary hormone deficiency syndromes. For example, rare syndromes of isolated ACTH, $\mathrm{TSH}$, or GH deficiency with unclear genetic causes could, in fact, be ascribed to defective hypothalamic $\mathrm{CRH}, \mathrm{GHRH}$, or TRH neuron development or function, respectively. The identification of these defects is a daunting task, as these syndromes are usually rare, making sporadic mutations difficult to identify and convincingly demonstrate to be the underlying cause of dysfunction. Moreover, these syndromes could likely turn out to be oligogenic disorders, as was the case for the individuals with Kallmann syndrome characterized by Cariboni and colleagues. Nevertheless, the successful application of novel techniques to unravel complex hormonal phenotypes exemplified by Cariboni et al. is encouraging for the elucidation of heretofore enigmatic molecular causes of central reproductive, adrenal, thyroid, and growth failure.

\section{Acknowledgments}

The author is grateful to Anat Ben-Shlomo, Ning-Ai Liu, and P. Michael Conn for critical reading of the manuscript and to Grace Labrado for manuscript preparation. Work in the author's laboratory is supported by NIH grant DK 103198 and the Doris Factor Molecular Endocrinology Laboratory.

Address correspondence to: Shlomo Melmed, Cedars-Sinai Medical Center, 8700 Beverly Blvd., Room 2015, Los Angeles, California 90048, USA. Phone: 310.423.4691; E-mail: melmed@csmc.edu. 
1. Melmed S. Mechanisms for pituitary tumorigenesis: the plastic pituitary. J Clin Invest. 2003;112(11):1603-1618.

2. Sykiotis GP, Pitteloud N, Seminara SB, Kaiser UB, Crowley WF Jr. Deciphering genetic disease in the genomic era: the model of GnRH deficiency. Sci Transl Med.2010;2(32):32rv2.

3. Semple RK, Topaloglu AK. The recent genetics of hypogonadotrophic hypogonadism - novel insights and new questions. Clin Endocrinol (Oxf). 2010;72(4):427-435.

4. Legouis R, et al. The candidate gene for the $\mathrm{X}$-linked Kallmann syndrome encodes a protein related to adhesion molecules. Cell. 1991;67(2):423-435.

5. Millar RP, Lu ZL, Pawson AJ, Flanagan CA, Morgan K, Maudsley SR. Gonadotropinreleasing hormone receptors. Endocr Rev. 2004;25(2):235-275.

6. Magill JC, Ciccone NA, Kaiser UB. A mathematical model of pulse-coded hormone signal responses in pituitary gonadotroph cells. Math Biosci. 2013;246(1):38-46.

7. Pitteloud N, Durrani S, Raivio T, Sykiotis GP. Complex genetics in idiopathic hypogonadotropic hypogonadism. Front Horm Res. 2010;39:142-153.

8. Kallmann FJ, Schoenfeld WA, Barrera SE. The genetic aspects of primary eunuchoidism. Am J Mental Deficiency. 1944;48:203-236.

9. Cariboni A, et al. The product of X-linked Kallmann's syndrome gene (KAL1) affects the migratory activity of gonadotropin-releasing hormone (GnRH)-producing neurons. Hum Mol Genet. 2004;13(22):2781-2791.

10. Costa-Barbosa FA, et al. Prioritizing genetic testing in patients with Kallmann syndrome using clinical phenotypes. J Clin Endocrinol Metab. 2013;98(5):E943-E953.

11. Cariboni A, et al. Dysfunctional SEMA3E signaling underlies gonadotropin-releasing hormone neuron deficiency in Kallmann syndrome. J Clin Invest. 2015;125(6):2413-2428.

12. Navarro VM, et al. The integrated hypothalamic tachykinin-kisspeptin system as a central coordinator for reproduction. Endocrinology. 2015;156(2):627-637.

13. Navarro VM, Kaiser UB. Metabolic influences on neuroendocrine regulation of reproduction. Curr Opin Endocrinol Diabetes Obes. 2013;20(4):335-341.

14. Stewart MD, et al. Mice harboring Gnrhr E90K, a mutation that causes protein misfolding and hypogonadotropic hypogonadism in humans, exhibit testis size reduction and ovulation failure. Mol Endocrinol. 2012;26(11):1847-1856.

15. Tao YX, Conn PM. Chaperoning G protein-coupled receptors: from cell biology to therapeutics. Endocr Rev. 2014;35(4):602-647.

16. Layman LC. Clinical genetic testing for Kallmann syndrome. JClin Endocrinol Metab. 2013;98(5):1860-1862.

17. Sato N, et al. Clinical assessment and mutation analysis of Kallmann syndrome 1 (KAL1) and fibroblast growth factor receptor 1 (FGFR1, or KAL2) in five families and 18 sporadic patients. JClin Endocrinol Metab. 2004;89(3):1079-1088.

18. Valdes-Socin H, Rubio Almanza M, Tome Fernandez-Ladreda M, Debray FG, Bours V, Beckers A. Reproduction, smell, and neurodevelopmental disorders: genetic defects in different hypogonadotropic hypogonadal syndromes. Front Endocrinol (Lausanne). 2014;5:109.

19. Dode C, et al. Loss-of-function mutations in
FGFR1 cause autosomal dominant Kallmann syndrome. Nat Genet. 2003;33(4):463-465.

20. Kim HG, et al. Mutations in CHD7, encoding a chromatin-remodeling protein, cause idiopathic hypogonadotropic hypogonadism and Kallmann syndrome. Am J Hum Genet. 2008;83(4):511-519.

21. Marcos S, et al. The prevalence of CHD7 missense versus truncating mutations is higher in patients with Kallmann syndrome than in typical CHARGE patients. JClin Endocrinol Metab. 2014;99(10):E2138-E2143.

22. Hanchate NK, et al. SEMA3A, a gene involved in axonal pathfinding, is mutated in patients with Kallmann syndrome. PLoS Genet. 2012;8(8):e1002896.

23. Abreu AP, Noel SD, Xu S, Carroll RS, Latronico AC, Kaiser UB. Evidence of the importance of the first intracellular loop of prokineticin receptor 2 in receptor function. Mol Endocrinol. 2012;26(8):1417-1427.

24. Bianco SD, Kaiser UB. The genetic and molecular basis of idiopathic hypogonadotropic hypogonadism. Nat Rev Endocrinol. 2009;5(10):569-576.

25. Kim J, Oh WJ, Gaiano N, Yoshida Y, Gu C. Semaphorin 3E-Plexin-D1 signaling regulates VEGF function in developmental angiogenesis via a feedback mechanism. Genes Dev 2011;25(13):1399-1411.

26. Sykiotis GP, et al. Oligogenic basis of isolated gonadotropin-releasing hormone deficiency. Proc Natl Acad Sci U S A. 2010; 107(34):15140-15144.

27. Matsumoto S, et al. Abnormal development of the olfactory bulb and reproductive system in mice lacking prokineticin receptor PKR2. Proc Natl Acad Sci U S A. 2006;103(11):4140-4145. 